\title{
Evaluation of the Utility of von Willebrand Factor Propeptide in the Differential Diagnosis of von Willebrand Disease and Acquired von Willebrand Syndrome
}

\author{
${ }^{1}$ Angelo Bianchi Bonomi Hemophilia and Thrombosis Center, \\ Fondazione IRCCS Ca' Granda Ospedale Maggiore Policlinico and \\ Luigi Villa Foundation, Milan, Italy \\ 2 Internal Medicine Unit, Azienda Provinciale per i Servizi Sanitari, \\ Trento, Italy \\ ${ }^{3}$ Department of Pathophysiology and Transplantation, Università \\ degli Studi di Milano, Milan, Italy
}

Francesca Stufano, MSc ${ }^{1}$ Marco Boscarino, MSc ${ }^{1}$ Paolo Bucciarelli, MD ${ }^{1} \quad$ Luciano Baronciani, MSc ${ }^{1}$ Alberto Maino, MD, PhD ${ }^{1,2}$ Giovanna Cozzi, MSc ${ }^{1}$ Flora Peyvandi, MD, PhD ${ }^{1,3}$

\begin{abstract}
Address for correspondence Flora Peyvandi, MD, PhD, Angelo Bianchi Bonomi Hemophilia and Thrombosis Center, Fondazione IRCCS Ca' Granda Ospedale Maggiore Policlinico and Luigi Villa Foundation, Milan, Italy (e-mail: flora.peyvandi@unimi.it).
\end{abstract}

Semin Thromb Hemost 2019;45:36-42.

\begin{abstract}
An increased von Willebrand factor propeptide (VWFpp) to VWF antigen (VWF:Ag) ratio (VWFpp/VWF:Ag) indicates an enhanced clearance of VWF. This finding has been described in von Willebrand disease (VWD) and in acquired von Willebrand syndrome (AVWS). A distinction between these two diseases, one congenital and the other acquired, is primarily based on family and personal history of bleeding. However, if this information is scanty, the diagnosis might be challenging due to the lack of an effective diagnostic biomarker. In this cross-sectional study, we assessed the ability of VWFpp/VWF:Ag for the differential diagnosis between VWD and AVWS. VWFpp/VWF:Ag was measured in a group of 153 patients ( 125 with VWD and 28 with AVWS). Most patients with AVWS and VWD showed an increased VWFpp/VWF:Ag, although to variable degrees. A marked increase of VWFpp/ VWF:Ag was mainly associated with the diagnosis of AVWS and VWD type 1 Vicenza. A receiver operating characteristic curve was used to identify the optimal cutoff of VWFpp/ VWF:Ag for discrimination of patients with a modestly increased (most VWD cases) versus

Keywords

- VWFpp

- propeptide

- von Willebrand disease

- acquired von Willebrand syndrome those with a markedly increased clearance (AVWS and VWD type 1 Vicenza), and this cutoff was identified at the value of 3.9 (sensitivity: 0.70 , specificity: 0.97 ). The ROC curve sorting from a logistic model containing VWFpp/VWF:Ag, age, and sex had an area under the curve (AUC) of 0.88 (95\% confidence interval: 0.80-0.95). A subsequent molecular evaluation discriminated VWD type 1 Vicenza from AVWS. In conclusion, VWFpp/VWF:Ag appears helpful to discriminate patients with a markedly increase VWF clearance (AVWS or VWD type 1 Vicenza) from those with a modestly increased clearance (most VWD patients).
\end{abstract}

von Willebrand factor (VWF) is synthesized as pre-pro-VWF in endothelial cells and megakaryocytes, where it undergoes posttranslational modifications, including signal peptide cleavage, C-terminal dimerization, glycosylation, sulfation, and N-terminal multimerization. ${ }^{1}$ The VWF propeptide
(VWFpp) is cleaved within the trans-Golgi, but it remains stored together with VWF in $\alpha$-granules (megakaryocytes) and Weibel-Palade bodies (endothelial cells). ${ }^{2}$ Upon endothelial-cell stimulation or injury, VWFpp is secreted into the circulation, together with VWF and circulates in published online June 18, 2018
Issue Theme Editorial Compilation VI; Guest Editors: Emmanuel J. Favaloro, PhD, FFSc (RCPA), and Giuseppe Lippi, MD.

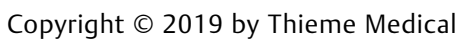
Publishers, Inc., 333 Seventh Avenue, New York, NY 10001, USA. Tel: +1(212) 584-4662.
DOI https://doi.org/ 10.1055/s-0038-1660481. ISSN 0094-6176. 
blood as a dimer with a 2-hour half-life. ${ }^{3}$ It has been reported that the steady state of VWFpp to VWF antigen (VWF:Ag) ratio (VWFpp/VWF:Ag) may be an indicator of degree of VWF clearance. ${ }^{4-6}$ An increased VWFpp/VWF:Ag indicates an enhanced VWF clearance, which has been described in both patients with von Willebrand disease (VWD) and acquired von Willebrand syndrome (AVWS). ${ }^{4-9}$

VWD, inherited in an autosomal dominant or recessive fashion, is the most common congenital bleeding disorder. Because different mutations can affect $V W F$, resulting in variable impact on circulating levels and activities of VWF, clinical symptoms of VWD vary from mild to severe. The classification of VWD in different forms reflects these heterogeneous causal mechanisms, and it is often challenging. ${ }^{10}$ Among VWD variants, VWD type 1 Vicenza is an extreme example of increased VWF clearance. Compared with healthy controls, the half-life of VWF type 1 Vicenza is shortened 4.4-fold after desmopressin, ${ }^{11}$ thus suggesting that rapid clearance of VWF accounts for the moderately severe plasma deficiency of this disease variant. VWD type 1 Vicenza is also characterized by proportionately low levels of VWF:Ag and VWF:RCo, ultra-large plasma VWF multimers, and normal platelet levels of VWF. ${ }^{12}$ Although low, VWF:Ag and VWF:RCo levels are decreased proportionately, ${ }^{13}$ so that VWF:RCo/VWF:Ag ratios are not reduced, and the Vicenza variant is therefore classified among VWD type 1.

AVWS is a rare, often underdiagnosed syndrome, characterized by quantitative, structural, or functional VWF defects that are acquired (i.e., not inherited), and often accompanied by a severe bleeding tendency. AVWS can be associated with different underlying diseases, including lymphoproliferative diseases, autoimmune disorders, and monoclonal gammopathies. ${ }^{14-16}$ The International Society of Thrombosis and Haemostasis (ISTH) registry reported that the highest frequency of AVWS occurs in elderly patients, with a median age at diagnosis of 62 years. ${ }^{17}$ Frequently, the mechanism responsible for AVWS is increased VWF clearance by autoantibodies. ${ }^{18}$ Because laboratory results in AVWS are similar to those in VWD, and include decreased plasma levels of VWF:Ag and VWF:RCo, it is often difficult to make a differential diagnosis between VWD and AVWS because the available diagnostic laboratory tests do not provide adequate information and the personal history of bleeding (acquired or lifelong) is not always conclusive. Therefore, a diagnostic biomarker may facilitate the differential diagnosis and subsequent management of these two disorders, both due to deficiency and dysfunction of VWF.

With this background and gaps of knowledge, the aim of this cross-sectional study was to evaluate the ability of VWFpp/VWF:Ag to differentiate VWD from the AVWS, either using this ratio alone or in combination with further laboratory evaluations.

\section{Patients and Methods}

\section{Patients}

A total of 153 nonconsecutive patients (125 with VWD and 28 with AVWS) were selected from our cohort referred for a bleeding diathesis to the Angelo Bianchi Bonomi Hemophilia and Thrombosis Center (Milan, Italy) between 1990 and 2017. The diagnosis of VWD and its classification were made according to the ISTH Scientific and Standardization Committee (SSC) guidelines. ${ }^{10}$ The personal and family history of bleeding, the decrease of VWF plasma levels, the VWF intraplatelet content, and the VWF multimeric pattern were used as diagnostic criteria of VWD. With the exception of VWD type 1, the diagnosis of all patients was confirmed at the molecular level by Sanger sequencing, as described elsewhere. ${ }^{19}$ AVWS was diagnosed with the same methods as for VWD, although also considering the new onset of a bleeding tendency with a previous negative personal history and presence of an underlying disease. ${ }^{20}$ The bleeding severity score (BSS) according to Tosetto et $\mathrm{al}^{21}$ was also collected for both VWD and AVWS at first visit in 125 of 153 patients to better evaluate personal bleeding history. All subjects gave their informed consent to participate in the study, which was approved by the Institutional Review Board of the Fondazione IRCCS Ca' Granda Ospedale Maggiore Policlinico.

\section{Laboratory Tests}

Blood samples for measurement of VWF:Ag and VWFpp were collected during routine follow-up visits, which took place between 2007 and 2017, in 3.2\% (0.105-0.109 mol/L) buffered citrate solution (1:9 anticoagulant to whole blood), centrifuged at $1,500 \mathrm{~g}$ for 15 minutes at room temperature, aliquoted and stored at $-80^{\circ} \mathrm{C}$ until assayed. For patients treated with FVIII/VWF concentrates or desmopressin, the last treatment was given at least 1 month before blood collection. VWF:Ag levels were measured with a homemade ELISA assay using polyclonal antibodies (Dako; A0082 and P0226), as previously described. ${ }^{22}$ VWFpp was measured with a commercially available ELISA kit (Sanquin), using antihuman VWFpp monoclonal antibodies. Normal laboratory reference ranges for VWF:Ag, VWFpp and VWFpp/VWF:Ag were determined as the 95th percentile distribution in 121 healthy blood donors (median age: 45 years, range: $21-64$ years): these were 51 to $165 \mathrm{IU} / \mathrm{dL}$ for VWF:Ag, 55 to $155 \mathrm{IU} / \mathrm{dL}$ for VWFpp, and 0.6 to 1.6 for VWFpp/VWF:Ag.

\section{Statistical Analysis}

Continuous variables were described as median with interquartile range (IQR), categorical variables as counts, and percentages. Because patients with VWD type 1 Vicenza and AVWS are characterized by a markedly increased clearance of VWF, our first step was to evaluate whether or not VWFpp/VWF:Ag could discriminate between patients with a modestly increased VWF clearance (most VWD cases) and those with a markedly increased VWF clearance (AVWS and type 1 Vicenza). For this purpose, in the frame of a logistic regression model, a receiver operating characteristic (ROC) curve was used to assess the optimal cutoff of VWFpp/VWF: $\mathrm{Ag}$ for their discrimination. This was identified as the value with the best compromise between sensitivity and specificity (Youden's index). An internal validation of the predictive model was performed with 1,000 bootstrap replicates to 
correct for optimism. The same (ROC) analysis was repeated after the exclusion of patients with AVWS secondary to essential thrombocythemia (ET), as these patients were found to comprise a special group of AVWS with non-high clearance. The discriminative ability of the full logistic model (containing VWFpp/VWF:Ag, sex, and age) was estimated as the area under the ROC curve (AUC), with its 95\% confidence intervals (CIs) also for VWD (type 1 Vicenza excluded) versus AVWS and for VWD (type 1 Vicenza excluded) versus type 1 Vicenza groups.

All statistical analyses were performed using the statistical software R, release 3.3.2 (R Foundation for Statistical Computing, Vienna, Austria).

\section{Results}

Baseline characteristics of the main patient groups are shown in -Table 1. VWD patients were categorized into type 1 Vicenza ( $n=14)$, VWD type 1 (Vicenza excluded; $n=33), 2$ A IIA ( $n=19), 2$ A IIE $(n=14), 2 \mathrm{~B}(n=25)$, and $2 \mathrm{M}$ $(n=20)$. - Table 2 shows the bioclinical patterns of patients with different VWD types. The VWFpp/VWF:Ag (median
[IQR]) was particularly high in patients with VWD type 1 Vicenza (10.4 [8.4-13.5]) and only moderately increased in VWD type 2 patients (2.3 [2.0-2.8]). - Table 3 describes the group of AVWS patients. The VWFpp/VWF:Ag (median [IQR]) was higher in patients with AVWS secondary to cardiovascular disease (9.6 [5.7-17.8]; $n=3$ ), lymphoproliferative disorders (8.0 [5.0-11.0]; $n=5$ ), and monoclonal gammopathies of undetermined significance (4.5 [4.1-8.9]; $n=12$ ), than in those with ET (1.4 [1.2-1.5]; $n=8)$. - Fig. 1 also shows that VWFpp/VWF:Ag values were higher in patients with AVWS and VWD type 1 Vicenza than in the remaining VWD patients.

Based on the ROC curve, the best cutoff value of VWFpp/ VWF:Ag for discriminating patients with a modestly increased VWF clearance (most VWD patients) versus those with a markedly increased VWF clearance (AVWS and VWD type 1 Vicenza) was 3.9, with a corresponding sensitivity of $0.70(95 \%$ CI: 0.55-0.81) and specificity of 0.97 (95\% CI: 0.93-0.99). The ROC curve sorting from the logistic model that contained VWFpp/VWF:Ag, age, and sex had an AUC of 0.88 (95\% CI: $0.80-0.95$; - Fig. 2A). After correction for optimism $(1,000$ bootstrap replicates), the AUC slightly decreased to 0.87. After

Table 1 Baseline characteristics of patients with VWD (type 1 Vicenza excluded), VWD type 1 Vicenza, AVWS, and AVWS (ET excluded)

\begin{tabular}{|l|l|l|l|l|}
\hline Variables & $\begin{array}{l}\text { VWD } \\
\text { (type 1 Vicenza } \\
\text { excluded) } \\
(\boldsymbol{n}=\mathbf{1 1 1})\end{array}$ & $\begin{array}{l}\text { VWD type 1 Vicenza } \\
(\boldsymbol{n}=14)\end{array}$ & $\begin{array}{l}\text { AVWS } \\
(\boldsymbol{n}=\mathbf{2 8})\end{array}$ & $\begin{array}{l}\text { AVWS } \\
\text { (ET excluded } \boldsymbol{n}=\mathbf{2 0})\end{array}$ \\
\hline Gender (male/female) & $42 / 69$ & $5 / 9$ & $15 / 13$ & $12 / 8$ \\
\hline Age at visit (y) & $26(13-42)$ & $45(22-62)$ & $56(43-71)$ & $63(55-72)$ \\
\hline Bleeding score $^{\mathrm{a}}$ & $10(4-16)$ & $17(9-19)$ & $10(3-14)$ & $12(7-16)$ \\
\hline VWF:Ag (IU/dL) & $35(22-46)$ & $10(9-13)$ & $34(15-65)$ & $21(14-35)$ \\
\hline VWFpp (IU/dL) & $63(46-92)$ & $99(89-122)$ & $116(89-143)$ & $123(101-150)$ \\
\hline VWFpp/VWF:Ag & $2.0(1.5-2.7)$ & $10.4(8.4-13.5)$ & $4.0(1.5-8.7)$ & $6.4(4.0-10.5)$ \\
\hline
\end{tabular}

Abbreviations: AVWS, acquired von Willebrand syndrome; VWD, von Willebrand disease; VWF, von Willebrand factor; VWF:Ag, VWF antigen; VWFpp, VWF propeptide; VWFpp/VWF:Ag, VWFpp to VWF:Ag ratio.

${ }^{a}$ Median values (interquartile range).

bormal range for VWFpp/VWF:Ag 0.6-1.6.

Table 2 Bioclinical patterns of the patients with different VWD variants

\begin{tabular}{|l|l|l|l|l|}
\hline Type of VWD & N (\%) & VWF:Ag $^{\mathbf{a}}$ (IU/dL) & $\begin{array}{l}\text { VWFpp } \\
\text { (IU/dL) }\end{array}$ & VWFpp/VWF:Ag $^{\mathbf{a}}$ \\
\hline 1 Vicenza & $14(11.2)$ & $10(9-13)$ & $99(89-122)$ & $10.4(8.4-13.5)$ \\
\hline 1 (Vicenza excluded) & $33(26.4)$ & $37(31-42)$ & $46(41-67)$ & $1.4(1.3-1.8)$ \\
\hline 2A IIA & $19(15.2)$ & $35(18-69)$ & $76(52-136)$ & $2.2(1.8-2.6)$ \\
\hline 2A IIE & $14(11.2)$ & $20(16-22)$ & $59(47-68)$ & $2.8(2.2-3.4)$ \\
\hline 2B & $25(20)$ & $46(35-53)$ & $104(90-127)$ & $2.4(1.8-2.9)$ \\
\hline 2M & $20(16)$ & $24(17-30)$ & $57(43-68)$ & $2.2(2.0-2.7)$ \\
\hline
\end{tabular}

Abbreviations: VWD, von Willebrand disease; VWF, von Willebrand factor; VWF:Ag, VWF antigen; VWFpp, VWF propeptide; VWFpp/VWF:Ag, VWFpp to VWF:Ag ratio.

${ }^{\mathrm{a}}$ Median values (interquartile range). 
Table 3 Bioclinical patterns of patients with AVWS

\begin{tabular}{|c|c|c|c|c|}
\hline Diagnosis & $N(\%)$ & VWF:Ag $(\mathrm{IU} / \mathrm{dL})$ & $\begin{array}{l}\text { VWFpp } \\
\text { (IU/dL) }\end{array}$ & VWFpp/VWF:Aga \\
\hline Cardiovascular disease & $3(11)$ & $16(12-25)$ & $154(108-168)$ & $9.6(5.7-17.8)$ \\
\hline Lymphoproliferative disorders $^{\mathrm{b}}$ & $5(18)$ & $15(14-23)$ & $118(104-120)$ & $8.0(5.0-11.0)$ \\
\hline MGUS & $12(43)$ & $25(14-35)$ & $126(104-144)$ & $4.5(4.1-8.9)$ \\
\hline Essential thrombocythemia & $8(28)$ & $74(68-105)$ & $97(85-117)$ & $1.4(1.2-1.5)$ \\
\hline All AVWS & $28(100)$ & $34(15-65)$ & $116(89-143)$ & $4.0(1.5-8.7)$ \\
\hline All AVWS (ET excluded) & $20(71)$ & $21(14-35)$ & $123(101-150)$ & $6.4(4.0-10.5)$ \\
\hline
\end{tabular}

Abbreviations: AVWS, acquired von Willebrand syndrome; ET, essential thrombocythemia; MGUS, monoclonal gammopathies of undetermined significance. VWD, von Willebrand disease; VWF, von Willebrand factor; VWF:Ag, VWF antigen; VWFpp, VWF propeptide; VWFpp/VWF:Ag, VWFpp to VWF:Ag ratio.

a Median values (interquartile range).

${ }^{b}$ Chronic lymphocytic leukemia, Waldenstrom's macroglobulinemia.

exclusion of the eight patients with AVWS secondary to ET (i.e., those with the lowest values of VWFpp/VWF:Ag who were found to represent a non-high clearance group), the best discriminative value of VWFpp/VWF:Ag was 3.5, with a sensitivity of 0.88 (95\% CI: 0.74-0.96), a specificity of 0.95 (95\% CI: 0.87-0.97), and an AUC of 0.96 (95\% CI: 0.91-1.00). The two ROC curves sorting from the logistic model that contained VWFpp/VWF:Ag, age, and sex as predictors for the discrimination of VWD (type 1 Vicenza excluded) from AVWS patients, and of VWD from type 1 Vicenza patients, are shown in - Fig. 2B and C, respectively.
The genetic analysis of the mutation at codon 1205 in the group with the markedly increased VWF clearance allowed us to distinguish between VWD type 1 Vicenza (characterized by the presence of the mutation p.R1205H) and AVWS (absence of this mutation).

\section{Discussion}

In this study, we measured VWFpp/VWF:Ag in 153 nonconsecutive patients with VWD or AVWS, to assess the potential diagnostic role of this marker in the differential diagnosis

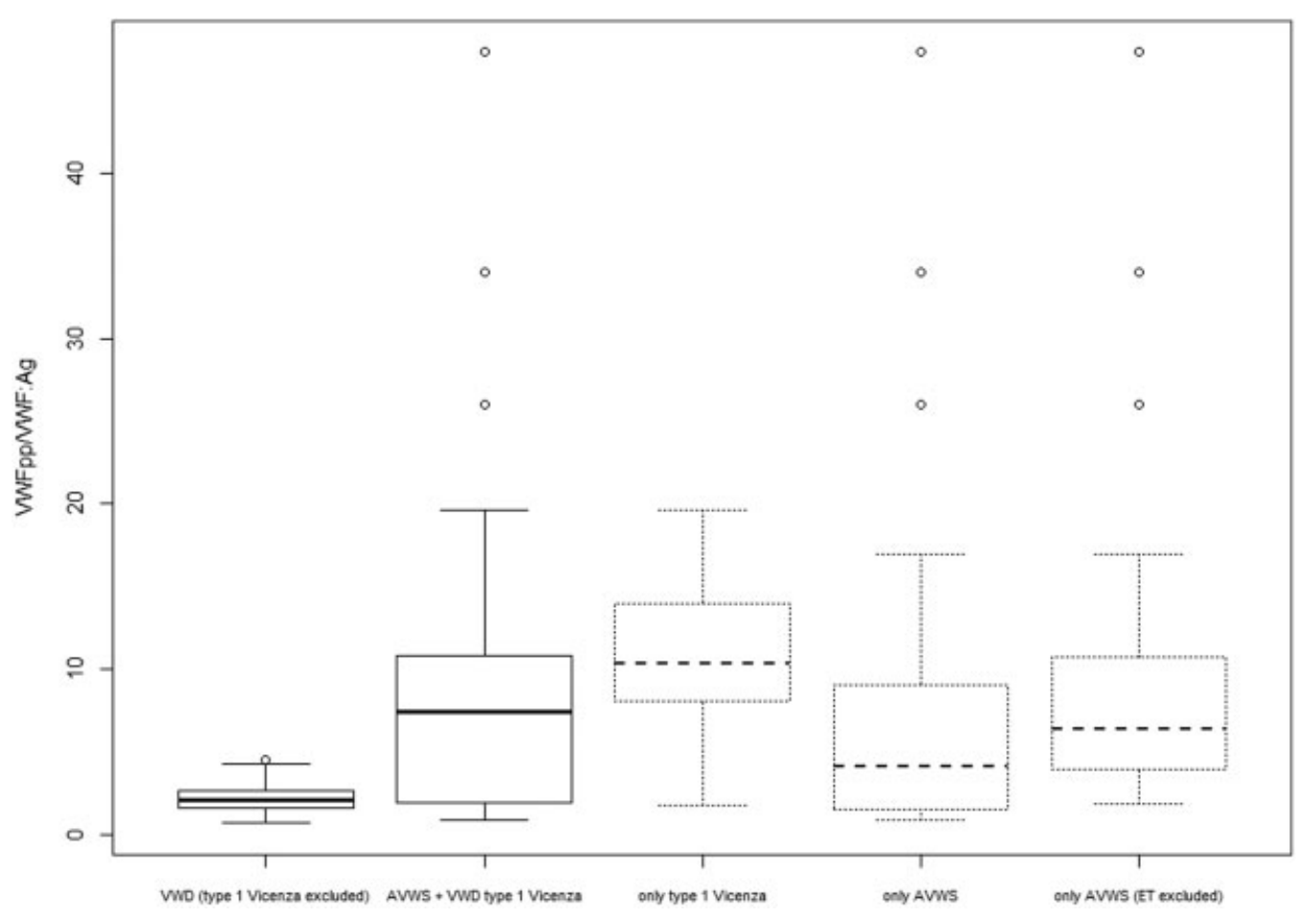

Fig. 1 Distribution of VWFpp/VWF:Ag values in VWD (type 1 Vicenza excluded), AVWS, and VWD type 1 Vicenza, only type 1 Vicenza, only AVWS, and only AVWS patients (essential thrombocythemia [ET] excluded). Each boxplot represents the interquartile range with median value (horizontal line). Open circles indicate outliers. Dotted boxplots represent type 1 Vicenza, AVWS patients, and AVWS patients (ET excluded) as separate groups. AVWS, acquired von Willebrand syndrome; VWD, von Willebrand disease; VWF, von Willebrand factor; VWF:Ag, VWF antigen; VWFpp, VWF propeptide; VWFpp/VWF:Ag, VWFpp to VWF:Ag ratio. 

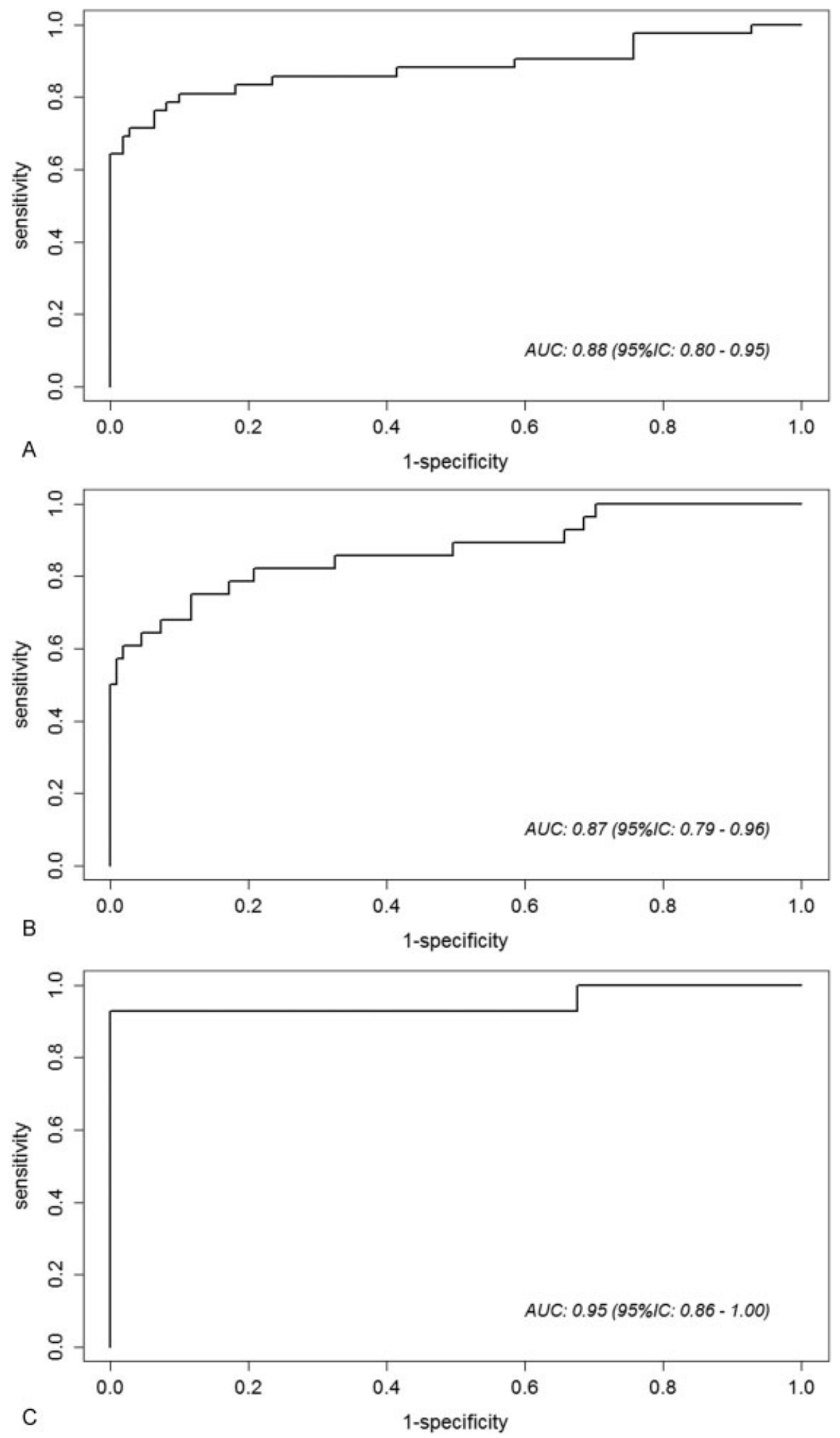

Fig. 2 Receiver operating characteristic (ROC) curves for discrimination of patients with modestly increased VWF clearance (most VWD) from those with markedly increased clearance (AVWS and type 1 Vicenza) (A), VWD (type 1 Vicenza excluded) vs. AVWS (B), and VWD vs. type 1 Vicenza (C). The ROC curves were obtained from a logistic model containing VWFpp/VWF:Ag, age, and sex. (A) VWD vs. AVWS + type 1 Vicenza. (B) VWD (type 1 Vicenza excluded) vs. AVWS. (C) VWD vs. type 1 Vicenza AVWS, acquired von Willebrand syndrome; VWD, von Willebrand disease; VWF, von Willebrand factor; VWF:Ag, VWF antigen; VWFpp, VWF propeptide; VWFpp/VWF:Ag, VWFpp to VWF:Ag ratio. 
between these two diseases. We found that VWFpp/VWF:Ag was markedly increased in cases characterized by higher VWF plasma clearance (i.e., AVWS and VWD type 1 Vicenza), as already reported. ${ }^{4-9}$ Most of the remaining VWD types, characterized by lower VWF clearance, invariantly had a VWFpp/VWF:Ag higher than the upper level of laboratory reference range (0.6-1.6), but generally less than those of AVWS and VWD type 1 Vicenza. Therefore, in the present study, we confirm the role of VWFpp/VWF:Ag as a tool for the diagnosis of both VWD and AVWS, because we found an increased value of this marker in the majority of patients included in our investigation. On the contrary, VWFpp/VWF: Ag was of little utility in the subclassification of other VWD types. Therefore, very high levels of the VWFpp/VWF:Ag should raise the suspicion of either AVWS or VWD type 1 Vicenza (i.e., the two groups of patients with the highest VWFpp/VWF:Ag in this study).

We also evaluated the potential diagnostic efficiency of VWFpp/VWF:Ag to discriminate patients with modestly increased VWF clearance (most VWD) from those with a markedly increased clearance (AVWS and VWD type 1 Vicenza). At values of 3.9 or higher, the VWFpp/VWF:Ag had a good discriminative power in identifying AVWS and VWD type 1 Vicenza, which was even better after excluding eight patients in the group of AVWS with the lowest VWFpp/VWF: $\mathrm{Ag}$ (i.e., those with ET; from an AUC of 0.88-0.96). The AUC of VWD (type 1 Vicenza excluded) versus AVWS and VWD versus type 1 Vicenza showed a good discriminative capability as well.

For the subsequent discrimination between AVWS and VWD type 1 Vicenza, we found that the genetic analysis for the identification of p.R1205H mutation in exon 27 of VWF was necessary, as shown in the present study. This study demonstrated that the good discrimination of patients with a markedly increased from those with a modestly increased VWF clearance based on the VWFpp/VWF:Ag values allowed the possibility to limit genetic analysis only to a minority of cases (one-third of patients in our study). Type Vicenza cases were confirmed by genetic analysis enabling the identification of both M740I (G2220A) and R1205H (G3614A) mutations in exons 17 and 27 of VWF.

This study has some limitations. As it included nonconsecutive patients, there is a possible relevant inherent selection bias. Second, the diagnostic utility of screening for VWFpp/VWF:Ag in consecutive patients with suspected VWD or AVWS has not yet been assessed, as this study included only selected patients with a confirmed diagnosis of VWD or AVWS. Second, we could not calculate the predictive values of our model, as they are influenced by disease prevalence, which could not be correctly estimated due to the study design. Another limitation is the small sample size of some subgroups of AVWS patients (i.e., cardiovascular disease and lymphoproliferative disorders subgroups).

\section{Conclusion}

VWFpp/VWF:Ag is a helpful tool to discriminate between patients with a markedly increased VWF clearance (AVWS or type 1 Vicenza) from those with a modestly increased clearance (most VWD patients), but molecular analysis is subsequently needed to finalize the differential diagnosis between AVWS and VWD type 1 Vicenza. Indeed, a markedly increased VWFpp/VWF:Ag will likely point to either VWD type 1 Vicenza or non-ET-associated AVWS. Larger studies with consecutive patient enrolment will be needed to confirm these preliminary findings.

\section{Authors' Contributions}

F.S., M.B., P.B., and L.B. wrote the article. A.M. critically revised the article. G.C. performed laboratory analysis. F.P. took part in the discussion and critically revised the article.

\section{Conflict of Interest}

F.P. has received honoraria for participating as a speaker at satellite symposia and educational meetings organized by Ablynx, Grifols, Sobi, Shire, F. Hoffmann-La Roche, and Alnylam. She is recipient of research grant funding from Ablynx, Novo Nordisk, Kedrion, and Biokit paid to Fondazione Luigi Villa, and she has received consulting fees from Kedrion and LFB. She is also member of the scientific advisory boards of Ablynx, F. Hoffmann-La Roche, and Shire. The other authors had no interests which might be perceived as posing a conflict or bias.

\section{Acknowledgments}

The authors would like to thank Prof. P.M. Mannucci for his critical advice and L.F. Ghilardini for the illustration work.

\section{References}

1 Wagner DD. Cell biology of von Willebrand factor. Annu Rev Cell Biol 1990;6:217-246

2 Ruggeri ZM. Von Willebrand factor, platelets and endothelial cell interactions. J Thromb Haemost 2003;1(07):1335-1342

3 Borchiellini A, Fijnvandraat K, ten Cate JW, et al. Quantitative analysis of von Willebrand factor propeptide release in vivo: effect of experimental endotoxemia and administration of 1deamino-8-D-arginine vasopressin in humans. Blood 1996;88 (08):2951-2958

4 Haberichter SL, Balistreri M, Christopherson P, et al. Assay of the von Willebrand factor (VWF) propeptide to identify patients with type 1 von Willebrand disease with decreased VWF survival. Blood 2006;108(10):3344-3351

5 Haberichter SL, Castaman G, Budde U, et al. Identification of type 1 von Willebrand disease patients with reduced von Willebrand factor survival by assay of the VWF propeptide in the European study: molecular and clinical markers for the diagnosis and management of type 1 VWD (MCMDM-1VWD). Blood 2008;111 (10):4979-4985

6 Sztukowska M, Gallinaro L, Cattini MG, et al. Von Willebrand factor propeptide makes it easy to identify the shorter Von Willebrand factor survival in patients with type 1 and type Vicenza von Willebrand disease. Br J Haematol 2008;143(01): 107-114

7 Schooten CJ, Tjernberg P, Westein E, et al. Cysteine-mutations in von Willebrand factor associated with increased clearance. J Thromb Haemost 2005;3(10):2228-2237

8 Eikenboom J, Federici AB, Dirven RJ, et al; MCMDM-1VWD Study Group. VWF propeptide and ratios between VWF, VWF 
propeptide, and FVIII in the characterization of type 1 von Willebrand disease. Blood 2013;121(12):2336-2339

9 van Genderen PJJ, Boertjes RC, van Mourik JA. Quantitative analysis of von Willebrand factor and its propeptide in plasma in acquired von Willebrand syndrome. Thromb Haemost 1998;80 (03):495-498

10 Sadler JE, Budde U, Eikenboom JCJ, et al; Working Party on von Willebrand Disease Classification. Update on the pathophysiology and classification of von Willebrand disease: a report of the Subcommittee on von Willebrand Factor. J Thromb Haemost 2006;4(10):2103-2114

11 Casonato A, Pontara E, Sartorello F, et al. Reduced von Willebrand factor survival in type Vicenza von Willebrand disease. Blood 2002;99(01):180-184

12 Mannucci PM, Lombardi R, Castaman G, et al. von Willebrand disease "Vicenza" with larger-than-normal (supranormal) von Willebrand factor multimers. Blood 1988;71(01):65-70

13 Castaman G, Rodeghiero F, Mannucci PM. The elusive pathogenesis of von Willebrand disease Vicenza. Blood 2002;99(11): 4243-4244, author reply 4244

14 Mannucci PM, Lombardi R, Bader R, et al. Studies of the pathophysiology of acquired von Willebrand's disease in seven patients with lymphoproliferative disorders or benign monoclonal gammopathies. Blood 1984;64(03):614-621
15 Castaman G, Lattuada A, Ruggeri M, Tosetto A, Mannucci PM, Rodeghiero F. Platelet von Willebrand factor abnormalities in myeloproliferative syndromes. Am J Hematol 1995;49(04): 289-293

16 Colella MP, Duarte GC, Marques JF Jr, De Paula EV. Haemostatic management of extreme challenges to haemostasis in acquired von Willebrand syndrome. Haemophilia 2012;18(03):e188-e191

17 Federici AB, Rand JH, Bucciarelli P, et al; Subcommittee on von Willebrand Factor. Acquired von Willebrand syndrome: data from an international registry. Thromb Haemost 2000;84(02):345-349

18 Kumar S, Pruthi RK, Nichols WL. Acquired von Willebrand disease. Mayo Clin Proc 2002;77(02):181-187

19 Baronciani L, Cozzi G, Canciani MT, et al. Molecular characterization of a multiethnic group of 21 patients with type 3 von Willebrand disease. Thromb Haemost 2000;84(04):536-540

20 Tiede A. Diagnosis and treatment of acquired von Willebrand syndrome. Thromb Res 2012;130(Suppl 2):S2-S6

21 Tosetto A, Castaman G, Rodeghiero F. Assessing bleeding in von Willebrand disease with bleeding score. Blood Rev 2007;21(02): 89-97

22 Federici AB, Canciani MT, Forza I, et al. A sensitive ristocetin cofactor activity assay with recombinant glycoprotein Ib alpha for the diagnosis of patients with low von Willebrand factor levels. Haematologica 2004;89(01):77-85 